\title{
Éditorial
}

\section{LE SCANDALE FINANCIER COMME PROJET DE RECHERCHE}

\author{
Malik Mazbouri, Thibaud Giddey, Patrice Baubeau
}

ESKA | «ntreprises et histoire »

2020/4 n 101 | pages 6 à 13

ISSN 1161-2770

ISBN 9782747231220

Article disponible en ligne à l'adresse :

https://www.cairn.info/revue-entreprises-et-histoire-2020-4-page-6.htm

Distribution électronique Cairn.info pour ESKA.

(C) ESKA. Tous droits réservés pour tous pays.

La reproduction ou représentation de cet article, notamment par photocopie, n'est autorisée que dans les limites des conditions générales d'utilisation du site ou, le cas échéant, des conditions générales de la licence souscrite par votre établissement. Toute autre reproduction ou représentation, en tout ou partie, sous quelque forme et de quelque manière que ce soit, est interdite sauf accord préalable et écrit de l'éditeur, en dehors des cas prévus par la législation en vigueur en France. Il est précisé que son stockage dans une base de données est également interdit. 


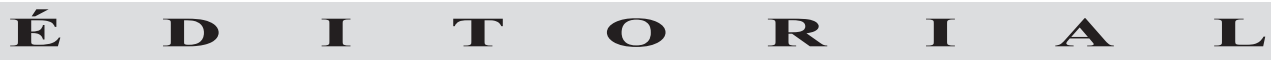 \\ LE SCANDALE FINANCIER \\ COMME PROJET DE RECHERCHE
}

\author{
par Malik MAZBOURI
}

maître d'enseignement et de recherche en histoire contemporaine

Université de Lausanne

\section{Thibaud GIDDEY}

maître d'enseignement et de recherche suppléant en histoire contemporaine

Université de Lausanne

\section{Patrice BAUBEAU}

IDHE.S, maître de conférences HDR d'histoire contemporaine

Université de Paris Nanterre

Pour de bonnes et de mauvaises raisons, et notamment à cause de ses relations supposées avec l'histoire événementielle et politique, pour ne pas dire sensationnaliste, le monde historien a longtemps tardé à donner suite aux appels à la recherche sur le scandale lancés (déjà en 1954) par Éric de Dampierre dans les Annales ${ }^{1}$. Certes, cette "réserve scientifique" ${ }^{2}$ a progressivement décru, à telle enseigne que nous disposons aujourd'hui d'une littérature académique importante et de qualité, qui a profondément renouvelé l'approche qu'on pouvait faire de ce phénomène social itératif et parfois tapageur qu'est le scandale ${ }^{3}$. Mais s'il est réjouissant de constater que, depuis quelques années, l'intérêt pour l'étude du scandale et des " affaires " est effectivement allé croissant, il convient aussi de noter que, à de rares exceptions près - en dépit même d'une brûlante actualité depuis les événements de 2007-2008 et la multiplication des leaks et autres papers -, la thématique du "I scandale financier " n'a pas vraiment retenu l'attention des historiens.

Le présent numéro d'Entreprises et Histoire voudrait remédier à cet état de fait en lançant, à son tour, un appel à développer les recherches sur cet objet riche et

\footnotetext{
${ }^{1}$ É. de Dampierre, « Thèmes pour l'étude du scandale », Annales ESC, vol. 9, n 3, 1954, p. 328-336.

${ }^{2}$ H.-E. Friedrich, « Literaturskandale. Ein Problemaufriss », in H.-E. Friedrich (Hg.), Literaturskandale, Francfort, Peter Lang, 2009, p. 8.

${ }^{3}$ Dans l'espace francophone, la publication, en 2005, du numéro de Politix intitulé « À l'épreuve du scandale » constitue un moment important de la légitimation du scandale comme objet d'étude.
} 
pluridimensionnel, à la croisée de l'histoire économique et financière, mais aussi de l'histoire sociale, politique et culturelle.

\section{PROBLÈMES DE DÉFINITIONS}

Que faut-il entendre, au juste, par scandale financier ? Comme l'écrit Damien de Blic ici même, la définition voire la simple description de ce dont il s'agit ne va pas de soi. C'est d'abord que, comme toute autre forme de scandale, le scandale «financier» (dans l'espace anglo-saxon on parlera plus volontiers de business scandal ou de corporate scandal ${ }^{4}$ ) implique toujours plusieurs univers sociaux, nullement confinés au seul monde de la finance ou de l'économie. Aucun scandale financier qui ne présente une dimension médiatique ou une phase judiciaire, voire des ramifications politiques : certains auteurs hésitent, du reste, en parlant de scandale « politico-financier $»^{5}$. Or ces étiquettes sont trompeuses et réductrices, tant il est vrai, comme le montre Hervé Rayner, que les scandales « sont d'abord et avant tout des jeux à acteurs multiples $»^{6}$. L'immixtion d'intervenants exogènes au monde de la finance constitue une condition de l'éclatement d'un scandale financier et ce sera précisément un enjeu fondamental, pour les milieux mis en cause, que de réussir à confiner ou non les débats et les éventuelles sanctions à l'interne de la profession (via, par exemple, les tribunaux arbitraux, de plus en plus courus), à les ramener à de pures questions techniques ou encore à se défausser de leurs responsabilités sur d'autres acteurs et secteurs. C'est enfin que le scandale financier porte souvent sur des processus complexes, dans lesquels la qualification même des faits en " scandale » (ou leur minimisation par les intéressés) participe de luttes sémantiques productrices de sens autant que d'effets et dont il faut se garder d'être dupe ${ }^{7}$.

Le débat du présent numéro qui réunit des universitaires, des journalistes et des praticiens de la finance illustre bien ces enjeux sémantiques et les conflits d'approche, entre, d'une part, une lecture " par l'acte», pour laquelle la qualification opérationnelle et juridique des actes commis joue un rôle central dans l'identification du scandale et, d'autre part, une lecture « par l'utilité sociale », qui mesure le scandale à l'écart entre l'utilité sociale de l'activité considérée et ses coûts collectifs, dont les crises ou les krachs sont les expressions les plus graves. La définition du scandale apparaît alors, inévitablement, même si c'est le plus souvent de manière implicite, comme un des éléments clés du scandale lui-même.

Depuis le travail d'Éric de Dampierre, la plupart des sociologues et historiens du scandale s'accordent sur le fait que l'une des conditions nécessaires à l'éclatement d'un scandale, qui n'est rien d'autre qu'une forme de mobilisation des opinions, est la dénonciation publique d'une transgression morale ou légale, supposée ou avérée. L'histoire des scandales financiers renvoie donc, par ce biais, à la question des normes et des valeurs et du rapport à l'argent. Il serait toutefois très réducteur de la confondre avec l'histoire de la corruption ou des crimes économiques, qu'elle peut certes contribuer à éclairer, mais dont elle doit impérativement être distinguée ${ }^{8}$.

\footnotetext{
${ }^{4}$ Pour une revue de la littérature récente produite dans le champ anglo-saxon de l'histoire des entreprises sur la fraude et le scandale financier, voir H. Van Driel, "Financial fraud, scandals, and regulation: A conceptual framework and literature review", Business History, vol. 61, n 8, 2018, p. 1259-1299.

${ }^{5}$ R. Rémond, « Scandales politiques et démocratie », Études. Revue de culture contemporaine, n 6,1972 , p. 862 ; J.-Y. Mollier, Le scandale de Panama, Paris, Fayard, 1991.

${ }^{6}$ H. Rayner, Dynamique du scandale : de l'affaire Dreyfus à Clearstream, Paris, Le Cavalier Bleu, 2007, p. 58 ; voir également l'introduction de sa thèse : H. Rayner, Les scandales politiques. L'opération "Mains propres » en Italie, Paris, M. Houdiard, 2005, p. 7-37.

${ }^{7}$ A. Garrigou, « Le boss, la machine et le scandale. La chute de la maison Médecin », Politix, n 17, 1992, p. 7-35.

${ }^{8}$ Voir par exemple O. Dard, J. I. Engels, A. Fahrmeir et F. Monier (dir.), Les coulisses du politique dans l'Europe contemporaine, t. 3 : Scandales et corruption à l'époque contemporaine, Paris, A. Colin, 2014.
} 
D'abord parce que dans le domaine financier ou économique, comme dans tout autre domaine de la vie sociale, des actes moralement répréhensibles voire délictueux, même avérés et publiquement dénoncés, ne provoquent pas à coup sûr un scandale. À l'inverse, un scandale peut parfaitement éclater sans qu'aucun des faits « scandaleux » qui font l'objet d'une dénonciation publique ne soit finalement avéré, pourvu que l'importance des mobilisations puisse faire croire à la réalité des actes transgressifs. En d'autres termes, de la même manière qu'une affaire politique peut conduire la personnalité mise en cause à un acte désespéré, un scandale financier peut en théorie provoquer la faillite d'une entreprise - voire une crise d'ampleur - et dans les deux cas indépendamment de la véracité des faits allégués. Contrairement à l'adage populaire, le scandale montre ainsi qu'il n'est pas toujours correct de croire qu'il n'y a pas de fumée sans feu : non seulement il peut parfaitement y avoir le feu sans que le brasier ne se signale par de la fumée (cas dans lequel une transgression avérée ne produit aucun scandale et n'est, à la limite, pas portée à la connaissance du public ou l'est, mais sans réaction notable de ce dernier), mais la fumée elle-même peut « provoquer» l'incendie, par exemple lorsque de fausses allégations sont portées par un scandale qui provoque une faillite ou une crise.

On comprend donc que l'analyse de ce phénomène multidimensionnel croise, combine et puise à plusieurs sources disciplinaires, aussi bien du côté de l'histoire des crises financières et de l'histoire des médias que de celui de la sociologie des mobilisations et de l'histoire des mentalités.

Dans les lignes qui suivent, nous voudrions proposer quelques pistes de réflexion autour de cet objet, tout en présentant brièvement les différentes contributions réunies dans ce numéro.

\section{RÔLE DES MÉDIAS ET PROXIMITÉS SOCIALES}

Une des conditions nécessaires à l'émergence d'un scandale, c'est-à-dire à la transformation de faits jugés scandaleux en une véritable affaire publique, implique la mobilisation d'une audience indignée qui déborde du cercle restreint des communications interpersonnelles pour engager, plus largement, des acteurs sociaux externes au secteur mis en cause. Un regard extérieur à la communauté, ou encore la désolidarisation d'un membre du groupe, agit souvent comme un des éléments déclencheurs et perturbateurs de l'ordre des choses, nourrissant la dynamique du scandale. La figure du lanceur d'alerte - whistleblower -, parfois présentée comme une incarnation de pratiques médiatiques récentes et dont la protection s'est institutionnalisée aux ÉtatsUnis au cours des quarante dernières années, reprend en réalité des formes de dénonciation bien plus anciennes. L'avènement, au cours du XIX ${ }^{\mathrm{e}}$ siècle, des médias de masse joue à cet égard un rôle tout à fait considérable, comme l'indique John B. Thompson, dans ce phénomène de décloisonnement et de publicisation, sans lequel le scandale, au sens moderne du terme, ne peut advenir ${ }^{9}$. Il est d'ailleurs significatif que l'ensemble des articles rassemblés dans ce numéro éclaire, à des degrés divers, l'importance de la presse dans la manifestation, la diffusion et la mise en visibilité des réactions indignées.

Rapporté à la question du scandale financier, ce phénomène de médiatisation suscite au moins trois réflexions.

En premier lieu, il serait intéressant d'interroger, de manière diachronique et comparative, la façon dont se déroule cette mise en visibilité médiatique du scandale financier : celle-ci rencontre-t-elle plus d'obstacles dans des centres d'affaires relativement petits et très intégrés, à l'exemple récent du Luxembourg ou, plus

${ }^{9}$ J. B. Thompson, Political Scandal: Power and Visibility in the Media Age, Cambridge, Polity Press, 2000, p. $31-59$. 
ancien, de la Suisse, où les élites financières, administratives et politiques entretiennent d'étroites relations et dans lesquels il existe un solide « consensus social $»{ }^{10}$ sur l'importance des activités financières pour l'économie du pays, que dans des structures plus larges, où le développement du secteur financier se heurte a priori à plus de résistances, alors que défections internes et mises en cause publiques y sont peut-être plus probables et sans doute moins risquées ? L'article de Benjamin Zenner, qui analyse notamment les réactions des médias luxembourgeois à l'écroulement en 1982 d'une banque italienne installée dans le Grand-Duché, développe cette piste de recherche en révélant le large consensus dont bénéficient les pratiques de la place financière luxembourgeoise et les difficultés de mobilisations, notamment par la presse, que cette configuration sociale engendre.

Deuxième réflexion : la proximité entre milieux financiers et milieux politiques a pu être désignée, par référence notamment aux grands scandales qui ont secoué l'histoire de la III $^{e}$ République en France, comme un terreau particulièrement favorable à l'éclatement de scandales ${ }^{11}$. Aujourd'hui, ces rapports de voisinage, qui semblent s'être renforcés, sont à la fois mieux décrits, notamment à travers la notion de conflit d'intérêt, et paradoxalement banalisés, alors même que plusieurs auteurs les désignent comme des facteurs de nature à favoriser le déclenchement de graves crises financières ${ }^{12}$. Dans quelle mesure ces évolutions compliquent-elles le travail d'alerte des médias, par ailleurs eux-mêmes parfois sous l'influence de ces mêmes milieux d'affaires ? Souvent des règles d'incompatibilité entre fonctions parlementaires et activités de conseil auprès d'entreprises sont adoptées mais demeurent pas ou peu appliquées alors que le personnel politique est ici à la fois juge et partie. Aujourd'hui, tandis que la presse et les médias subissent de fortes pressions économiques, dans quelle mesure les réseaux sociaux et autres blogs d'information plus ou moins militants prennent-ils leur relais en offrant un espace pour les mobilisations et scandales financiers « digitaux »?

Enfin, le rôle de la presse spécialisée, qui attend encore ses historiens, mérite une attention particulière. En effet, il n'est pas rare que les journalistes financiers ne se cantonnent pas à une fonction de " mise en visibilité » de faits jusqu'alors dissimulés. C'est plutôt l'inverse qui semble se dégager de l'analyse rétrospective. Publicité tapageuse et mensongère en faveur d'une entreprise dont les hommes liges arrosent les rédactions, chantages à la fausse rumeur en échange de commandes publicitaires, silences intéressés : la presse financière a souvent été à la fois sujet, actrice et vectrice du développement du scandale ${ }^{13}$. Une analyse de son rôle dans le déclenchement ou l'étouffement de certains scandales financiers, jusqu'à la lecture qu'elle peut proposer de tel ou tel épisode scandaleux, permettrait, peut-être, de dépasser la simple thématique de la « vénalité » de la presse financière pour examiner la manière dont évoluent ses liens avec le monde des affaires et les représentations idéologiques dominantes de son temps ${ }^{14}$. Cette dimension est au cœur de la contribution à ce numéro

\footnotetext{
${ }^{10} \mathrm{Au}$ sens de H. G. Schröter, "Cartelization and Decartelization in Europe, 1870-1995: Rise and Decline of an Economic Institution”, Journal of European Economic History, vol. 25, n 1, 1996, p. 131.

${ }^{11}$ R. Rémond, « Scandales politiques », art. cit.

${ }^{12}$ S. Johnson et J. Kwak, 13 Bankers: the Wall Street Takeover and the Next Financial Meltdown, New York, Pantheon Books, 2010.

13 J.-N. Jeanneney, L'Argent caché : milieux d'affaires et pouvoirs politiques dans la France du XXe siècle, Paris, Fayard, 1981, en particulier p. 215-230 ; M. Martin, " Les débuts de la presse financière et la détermination de ses fonctions dans la France du XIX ${ }^{\mathrm{e}}$ siècle ", in L'Information financière, facteur d'interdépendance économique, Bruxelles, Bruylant, 1983, p. 112-142 ; J. Taylor, "Financial crises and the birth of the financial press, 1825-1880", in S. Schifferes et R. Roberts (eds.), The Media and Financial Crises: Comparative and Historical Perspectives, New York, Routledge, 2014, p. 203-214.

${ }^{14}$ M. Martin, « Retour sur "l'abominable vénalité de la presse française” », Le Temps des médias, n 6, 2006, p. 22-33.
} 
de deux jeunes chercheurs, Miguel Ortiz et German Laverde, qui explorent une facette encore peu connue d'un scandale financier emblématique, l'affaire de Panama (18791893). En ayant recours à une méthodologie économétrique, l'article mesure l'impact sur la valeur boursière de la compagnie mise en cause d'une couverture médiatique élogieuse par une presse corrompue. Ici comme dans de nombreux autres scandales financiers, le rôle des médias va bien au-delà de la simple caisse de résonance de mobilisations sociales.

Enfin, du point de vue méthodologique, il faut souligner à la fois l'apport pour les historiens de bases de données en ligne donnant accès à des titres de presse numérisés et leur moteur de recherche employant la reconnaissance optique de caractères (citons par exemple retronews.fr, britishnewspaperarchiv.co.uk, e-newspaperarchives.ch, impresso-project.ch, etc.), mais également les biais statistiques que l'utilisation exclusive de ces plates-formes peut induire ${ }^{15}$. Ces bases numérisées permettent par exemple de caractériser précisément le profil chronologique d'un scandale et de confronter nos catégories générales - crises, scandales, finance - aux perceptions et au vocabulaire des contemporains : c'est ainsi que la perception des scandales financiers comme une menace pour l'épargne (conception des contemporains) plutôt que comme un risque systémique pesant sur l'économie (analyse rétrospective des historiens) a conditionné non seulement la réception, mais encore la réponse politique et règlementaire des autorités et du Parlement français dans les années 1930, ce qui explique largement le « retard » français en matière de régulation bancaire, comme le montre la contribution de Patrice Baubeau et Angelo Riva.

\section{JEUX D'ÉCHELLES : ENTRE LE LOCAL, LE NATIONAL ET L'INTERNATIONAL}

Comme on le verra dans ce numéro, le scandale met en jeu une multitude d'acteurs dont le rayon d'activité peut beaucoup varier. Le scandale financier, en particulier, constitue, au moins depuis le XVIII ${ }^{\mathrm{e}}$ siècle, un phénomène qui dépasse souvent les frontières nationales. Une même affaire peut, en effet, avoir différentes répercussions à l'échelle locale, nationale ou internationale. La faillite de la banque Herstatt en 1974, un établissement allemand de moyenne importance basé à Cologne, donne par exemple l'impulsion initiale à l'adoption de nouvelles mesures de prévention dans le cadre du Comité de Bâle qui s'imposeront comme des normes standards internationales ${ }^{16}$. Il convient donc de s'interroger avec une certaine prudence sur l'échelle d'analyse la plus pertinente. De même que la disparition d'un établissement bancaire à vocation régionale et locale peut tout à fait trouver son origine dans des facteurs structurels internationaux (éclatement d'une bulle spéculative mondiale, investissements internationaux risqués, etc.), la faillite d'un poids lourd de la finance internationale, avec son potentiel de déstabilisation, peut avoir des répercussions dramatiques à une échelle très locale. La thématique du scandale financier invite donc à déplacer la focale d'observation du micro vers le macro - et inversement - afin de mieux cerner les différents théâtres du déploiement et de la résolution d'une affaire.

Cette interrogation sur les différents théâtres de déploiement d'un scandale est soulevée à bon escient dans la contribution

\footnotetext{
${ }^{15}$ Pour un aperçu de ces interfaces et de leur utilisation possible par les chercheurs, cf. M. Ehrmann, E. Bunout, M. Düring, "Historical Newspaper User Interfaces: a Review", in IFLA WLIC 2019 - Athens, Greece - Libraries: dialogue for change, 2019 ; voir également H. Bødker, "Journalism History and Digital Archives", Digital Journalism, vol. 6, n 9, 2018, p. 1113-1120.

${ }^{16}$ E. Mourlon-Druol, “'Trust is good, control is better': The 1974 Herstatt Bank Crisis and its Implications for International Regulatory Reform”, Business History, vol. 57, n 2, 2015, p. 311-334.
} 
à ce numéro de Benoît Majerus. Il y retrace les soubresauts au Luxembourg d'une fraude financière d'ampleur internationale, la faillite du fonds d'investissement Investors Overseas Services dans les années 1970. La fraude pyramidale, qualifiée de " scandale » dans la presse internationale, connaît une couverture médiatique bien moins tapageuse au Luxembourg, lieu de domiciliation du fonds d'investissement. On y retrouve aussi la réflexion sur les possibilités d'émergence d'une mobilisation dans un petit pays marqué par de fortes proximités sociales.

Dans la perspective d'une sociologie historique soucieuse de comprendre le comportement des agents, il serait également pertinent de savoir si les différentes phases d'internationalisation/renationalisation qui ont marqué l'histoire mondiale de la finance ont modifié ou non la façon dont les acteurs eux-mêmes appréhendent l'inscription géographique et la portée de leurs agissements. Quid, à cet égard, du rôle des grandes organisations financières internationales ? On pense notamment au Comité financier de la Société des Nations après la Première Guerre mondiale, mais aussi à la Banque des Règlements internationaux, au FMI, au Club de Paris, ou encore au Comité de Bâle sur le contrôle bancaire. Au-delà de leurs fonctions officielles d'instances supranationales de contrôle ou de régulation, on pourrait aborder ces institutions comme des dispositifs visant autant à résoudre certaines crises qu'à éviter que celles-ci ne dégénèrent, le cas échéant, en scandales d'ampleur, susceptibles de remettre en cause la légitimité des systèmes financiers. La même approche pourrait valoir, au niveau national, pour des institutions de type banque centrale, trop souvent comprises à partir de leurs mandats techniques (conduire la politique monétaire, veiller à la stabilité des prix, etc.) dans une perspective fonctionnaliste, faisant l'impasse sur ce qui s'y joue, également, en termes politiques et sociétaux.

\section{MOBILISATIONS ET DÉMOBILISATIONS : PHASE JUDICIAIRE ET RÉSOLUTION DES SCANDALES}

Il serait évidemment naïf de confondre les faits scandaleux, qui suscitent une forme d'indignation, avec les infractions juridiques auxquelles elles peuvent (ou non) correspondre. Le traitement d'une affaire par la justice, s'il a lieu, constitue toutefois une étape importante dans l'analyse d'un scandale. Le déplacement d'une affaire vers un environnement dont les codes sont objectivement normés rend parfois possible une prise de distance avec les acteurs impliqués. Alors que les récits autour desquels se structurent les scandales par presse interposée et les prises de position publiques des intervenants donnent lieu à une alternance des rôles endossés - les coupables dénoncés se présentant en victimes de calomnies -, le traitement judiciaire opère une clarification des responsabilités. Il autorise une identification des diverses parties prenantes (stakeholders). De plus, par le travail d'enquête et d'instruction mené, le passage par l'arène judiciaire signifie aussi souvent une forme de publicisation des transgressions mises en cause - autant de traces qui intéresseront l'historien. Dans le cas du scandale financier, le traitement par la justice révèle souvent une forte asymétrie entre la gravité des infractions commises (du moins mesurée à l'importance des montants concernés), d'une part, et le degré d'attention du public et les sanctions auxquelles s'exposent les transgresseurs, d'autre part. La gestion judiciaire ou extra-judiciaire des illégalismes fiscaux, par exemple, constitue un paramètre essentiel dans le traitement de ce type de délinquance ${ }^{17}$. Enfin, il ne faut pas non plus négliger le rôle résolutoire que joue la phase judiciaire d'un

\footnotetext{
${ }^{17}$ A. Amicelle et J. Bérard, « Défense des classes dominantes : la division du travail de légitimation à l'épreuve des scandales financiers internationaux ", Revue de la régulation. Capitalisme, institutions, pouvoirs, $\mathrm{n}^{\circ} 22,2017$, p. 1-29.
} 
scandale, qui permet de mettre un terme à l'indignation publique et favorise le retour à l'ordre. La sanction pénale revient alors à clore le débat : les fautifs individuels ayant été éventuellement punis (on parle très volontiers de boucs émissaires ${ }^{18}$ ), tandis que les facteurs systémiques ayant permis le développement des délits demeurent inchangés, quand bien même les responsables de ceux-ci se seraient prévalus de ceux-là pour se blanchir des fautes commises. Le document présenté dans ce numéro par Johanna Gautier, à savoir la campagne médiatique de soutien à Mike Milken, le « roi des obligations pourries » condamné pour fraude et délit d'initié à la fin des années 1980, montre bien comment certains réduisent le scandale aux figures individuelles, tantôt bourreau, tantôt martyr.

L'article du présent numéro consacré aux crises et aux scandales financiers en Suisse insiste notamment sur cette phase de résolution des affaires. Malik Mazbouri et Thibaud Giddey, en s'interrogeant sur la faiblesse des mobilisations et des indignations collectives à l'échelle nationale, soulignent la remarquable aptitude des milieux bancaires helvétiques à éteindre rapidement les incendies déclenchés par les événements dénoncés. À la désignation expiatoire de quelques boucs émissaires individuels succède un prompt retour au business as usual qui s'opère en limitant la portée du scandale à un cadre institutionnalisé.

\section{LA QUESTION DES VALEURS : L'EXEMPLE DU RAPPORT À L'ARGENT}

Le scandale financier ou politico-financier est une figure classique du scandale qui révèle souvent l'écart existant entre les normes professionnelles revendiquées par les milieux intéressés et leurs pratiques réelles. Hautement mobilisateur, voire déstabilisateur, sur certaines périodes (fin du XIX ${ }^{\mathrm{e}}$ siècle, entre-deuX-guerres, années 1970), il paraît l'être beaucoup moins à partir de la fin du $\mathrm{XX}^{\mathrm{e}}$ siècle. Certains auteurs comme Damien de Blic s'interrogeaient, il y a vingt ans déjà, sur la question de savoir si la difficulté de mobiliser autour du « scandale » du Crédit lyonnais au début des années 1990 ne tenait pas à la technicité intrinsèque du dossier, à la difficulté de profiler un coupable dans les chaînes de responsabilités et à trouver des victimes ${ }^{19}$. La perte d'efficacité de ce type de scandale comme arme de mobilisation politique trahit-elle une perte du rapport social critique à l'argent ? Damien de Blic revisite cette question dans le présent numéro en réinscrivant le scandale du Crédit lyonnais dans une séquence d'interprétation plus longue (1994-2020). La prise en compte du contexte social postérieur à la crise de 2008 , marqué par la montée en puissance d'un discours critique à l'égard des banques et du crédit, permet de compléter les transformations successives du paysage normatif relatif à l'argent et à la finance ${ }^{20}$.

La contribution à ce numéro de Jean-Yves Mollier, éminent spécialiste du scandale de Panama, explore également la question du scandale en tant que vecteur de mobilisation. Intervenant au terme d'une série de krachs retentissants, il permet la diffusion d'un discours antiparlementaire et antisémite, notamment porté par la droite boulangiste. $\mathrm{La}$ révélation du trafic d'influence des hommes politiques impliqués ébranle profondément les institutions, tandis que des boucs émissaires, symétrique inversée des bons Français qu'ils ont abusés, sont rapidement désignés à la vindicte publique : le scandale de Panama prélude directement à l'affaire Dreyfus et participe

\footnotetext{
${ }^{18}$ Voir par exemple P. H. Hansen, « Making Sense of Financial Crisis and Scandal: A Danish Bank Failure in the First Era of Finance Capitalism », Enterprise \& Society, vol. 13, n 3, 2012, p. 672706.

${ }^{19}$ D. de Blic, «"Le scandale financier du siècle, ça ne vous intéresse pas ?”. Difficiles mobilisations autour du Crédit lyonnais », Politix. n 52, 2000, p. 157-181.

${ }^{20}$ M. Feher, Le temps des investis. Essai sur la nouvelle question sociale, Paris, La Découverte, 2017.
} 
à la construction, sur le long terme, d'un récit qui, tout en épargnant certaines grandes figures nationales, permet de discréditer la $\mathrm{III}^{\mathrm{e}}$ République et ses élites.

\section{RÔLE DES SCIENCES SOCIALES DANS LA FABRICATION DU SCANDALE}

Dès la fin du XIX ${ }^{\mathrm{e}}$ siècle, les sciences sociales et humaines sont très impliquées dans la «fabrication de sens » des faits sociaux et la production de savoirs sur l'État et la société ${ }^{21}$. Qu'il s'agisse d'historiens, d'économistes, de sociologues ou de politistes, ces « conseiller[s] collectif[s] du prince $»^{22}$, comme disait Michael Pollak, interviennent en tant qu'experts en légitimant ou en disqualifiant les qualificatifs posés par d'autres acteurs. En relisant le passé, les historiens, souvent avec des lunettes fortement imprégnées par des regards contemporains et leurs propres valeurs, peuvent transformer des faits sociaux, jugés à l'époque comme non scandaleux, voire tout simplement ignorés, en scandale. C'est le cas de l'excellente étude de Jean Bouvier sur le scandale de Panama, qui postule, a posteriori, deux scandales : l'affaire du canal transocéanique, telle qu'elle éclate en novembre 1892 suite au suicide du banquier de Reinach, et l'autre scandale, invisible aux yeux du public, relatif au rôle des banques dans la débâcle, les marges " scandaleuses " qu'elles se sont octroyées dans les opérations de financement de l'entreprise n'étant pas étrangères à la déconfiture financière de l'entreprise ${ }^{23}$. Quoi qu'on puisse penser des marges et de la responsabilité financière effective des banques dans l'éclatement du scandale de Panama, celles-ci, comme le souligne Jean-Yves Mollier dans son article, ont été peu mises en cause à l'époque. Jean Bouvier en convient du reste lui-même et on a là l'exemple d'un scandale en quelque sorte forgé par le chercheur lui-même, qui plaque par projection dans le passé ses propres valeurs sur l'objet analysé, confondant en partie le fait scandaleux avec la mobilisation que le dévoilement d'un tel fait aurait pu (ou dû) provoquer.

Ces interrogations sur les valeurs qui viennent nourrir un scandale et sa dénonciation nourrissent le « clin d'œil » de Flore Di Sciullo, qui se demande ce que peut signifier le scandale si on l'interroge dans le contexte de l'art contemporain. Son ampleur est certes moindre que le scandale financier tel qu'il est le plus souvent entendu dans ce numéro, mais son ancrage dans les arènes médiatiques et juridiques mérite comparaison.

Le scandale, de quelque nature qu'il soit, est un objet d'analyse passionnant, mais aussi un miroir déformant, dont l'historien doit interroger le reflet avec la plus grande prudence ; pour les raisons qu'on vient de signaler, il n'est, dans aucun domaine de la vie sociale, un baromètre de la moralité publique des contemporains. Déplorer la dégradation croissante de la déontologie des milieux d'affaires en raison de la multiplication des scandales financiers serait donc aussi naïf que de se féliciter de la progression de leur moralité du seul fait de la raréfaction de tels scandales. Puisse le présent numéro d'Entreprises et Histoire contribuer à stimuler les travaux sur ce phénomène social et historique éminemment transdisciplinaire et trop négligé par la recherche.

\footnotetext{
${ }^{21}$ P. Wagner, Sozialwissenschaft und Staat : Frankreich, Italien, Deutschland 1870-1980, Francfort-New York, Campus Verlag, 1990 ; R. Graf, K. C. Priemel, «Zeitgeschichte in der Welt der Sozialwissenschaften. Legitimität und Originalität einer Disziplin », Vierteljahrshefte für Zeitgeschichte, vol. 59, n 4, 2011, p. 479-508.

${ }^{22}$ M. Pollak, «Un texte dans son contexte. L'enquête de Max Weber sur les ouvriers agricoles », Actes de la recherche en sciences sociales, $\mathrm{n}^{\circ} 65,1986, \mathrm{p} .70$.

${ }^{23}$ J. Bouvier, Les deux scandales de Panama, Paris, Julliard, 1964.
} 